\title{
O015: A novel antibacterial material for transparent dressings
}

\author{
W Zingg ${ }^{1 *}$, L Clack ${ }^{1}$, C Ginet ${ }^{1}$, H Ney ${ }^{2}$, G Renzi ${ }^{3}, M$ Black ${ }^{4}$, M Martin $^{5}$ \\ From 2nd International Conference on Prevention and Infection Control (ICPIC 2013) \\ Geneva, Switzerland. 25-28 June 2013
}

\section{Introduction}

Intravascular lines are indispensable in hospital care. The main complication of their use is catheter-related bloodstream infection (CRBSI). Transparent, semipermeable dressings (TSD)are standard in covering the insertion site. 'Bionate' is a polymer with antibacterial properties and yet has not been taken up by a company to be marketed as a medical product. The polymer backbone consists of polyurethane while the surface consists of self-assembling monolayer end groups of quaternary ammonium structures. These structure properties make the material a candidate to produce easy-touse antibacterial TSD.

\section{Objectives}

The aim of the study was to assess the effectiveness of the material as a growth inhibitor of microorganisms on the skin of healthy volunteers.

\section{Methods}

Sterile 'Bionate' and control patches were applied to undisinfected skin (upper arm) of 10 healthy volunteers for 3 , 5 , and 7 days. Five volunteers tested the patch for 10 days. After removal, a sterile, moistened cotton swab was taken from the skin site and the patches were put into normal sterile saline. Skin swabs were transferred into $3 \mathrm{ml}$ sterile saline and incubated for 2 minutes at room temperature. Patches in saline were vortexed and incubated for at least 15 minutes at room temperature. Dilution series were prepared and 100ul of each probe were put onto trypticasesoy-agar for growth and colony counts. Agar plates were incubated at $35^{\circ} \mathrm{C}$ for up to $48 \mathrm{~h}$ and colony forming units (CFU) were counted thereafter.

\section{Results}

Log growth differences of skin swabs between 'Bionate' and control patches on days $3,5,7$, and 10 were 1.4, 2.5, 2.0 , and 1.4, respectively. Growth difference was significant from day 7 onwards $(P=0.002)$. Log growth differences of the 'Bionate' and control patches on days 3, 5, 7, and 10 were $1.8,1.8,2.5$, and 1.9 , respectively. Growth reduction was significant from day 5 onwards $(\mathrm{P}=0.015)$.

\section{Conclusion}

The study identified remarkable growth of microorganisms on skin covered with polyurethane dressings. Growth reduction of 'Bionate' as compared to standard polyurethane makes this material promising to develop a future TSD with inherent antimicrobial properties.

\section{Disclosure of interest}

None declared.

\section{Author details}

${ }^{1}$ Infection Control Program, University of Geneva Hospitals, Geneva, Switzerland. ${ }^{2}$ Department of Sterilisation, University of Geneva Hospitals, Geneva, Switzerland. ${ }^{3}$ Molecular Biology Lab, University of Geneva Hospitals, Geneva, Switzerland. ${ }^{4}$ Palm Beach Children's Hospital, Palm Beach, USA. ${ }^{5}$ Infection control program, Regionale Gesundheitsholding HeilbronnFranken, Helibronn, Germany.

Published: 20 June 2013

doi:10.1186/2047-2994-2-S1-015

Cite this article as: Zingg et al:: 0015: A novel antibacterial material for transparent dressings. Antimicrobial Resistance and Infection Control 2013 2(Suppl 1):015.

${ }^{1}$ Infection Control Program, University of Geneva Hospitals, Geneva,

Switzerland

Full list of author information is available at the end of the article

(c) 2013 Zingg et al; licensee BioMed Central Ltd. This is an Open Access article distributed under the terms of the Creative Commons Attribution License (http://creativecommons.org/licenses/by/2.0), which permits unrestricted use, distribution, and reproduction in any medium, provided the original work is properly cited. 